\title{
A Phase Transition and a Quadratic Time Unbiased Estimator for Network Reliability
}

\author{
David R. Karger \\ MIT \\ Computer Science and AI Laboratory,, Cambridge,, MA., USA \\ karger@mit.edu
}

\begin{abstract}
We improve the time for approximating network (un)reliability to $\tilde{O}\left(n^{2}\right)$. We do so not with a new algorithm, but with a deeper analysis and tweaking of algorithms from our previous work. In particular, we show that once a graph's failure probability shrinks below $1 / 2$, the graph rapidly transitions to a regime where even the expected number of cut failures is small, and in fact is almost exactly the same as the graph's failure probability. That is, we are very unlikely to ever see more than one cut fail. This lets us treat these cut failures as essentially independent, making it easier to estimate their likelihood. The contribution of this paper is not just the improved time bound, but also this clearer understanding of the evolution of a graph's reliability. Our results rely on some new methods for analyzing the distribution of cut failures conditioned on the failure of a particular cut, as well as new insights into the evolution of a graph's connectivity as edges are randomly added over time. Some of our results apply more broadly, to all monotone reliability systems.
\end{abstract}

\section{CCS CONCEPTS}

- Theory of computation $\rightarrow$ Random network models; Stochastic approximation; Routing and network design problems; Network flows; • Mathematics of computing $\rightarrow$ Random graphs; Paths and connectivity problems.

\section{KEYWORDS}

network reliability, unbiased estimator, randomized approximation scheme

\section{ACM Reference Format:}

David R. Karger. 2020. A Phase Transition and a Quadratic Time Unbiased Estimator for Network Reliability. In Proceedings of the 52nd Annual ACM SIGACT Symposium on Theory of Computing (STOC '20), fune 22-26, 2020, Chicago, IL, USA. ACM, New York, NY, USA, 11 pages. https://doi.org/10. $1145 / 3357713.3384336$

\section{INTRODUCTION}

Given a graph $G$, the (un)reliability $u_{G}(p)$ is the probability that $G$ becomes disconnected when every edge fails independently with

Permission to make digital or hard copies of all or part of this work for personal or classroom use is granted without fee provided that copies are not made or distributed for profit or commercial advantage and that copies bear this notice and the full citation on the first page. Copyrights for components of this work owned by others than the author(s) must be honored. Abstracting with credit is permitted. To copy otherwise, or republish, to post on servers or to redistribute to lists, requires prior specific permission and/or a fee. Request permissions from permissions@acm.org.

STOC '20, June 22-26, 2020, Chicago, IL, USA

(C) 2020 Copyright held by the owner/author(s). Publication rights licensed to ACM ACM ISBN 978-1-4503-6979-4/20/06.

https://doi.org/10.1145/3357713.3384336 probability $p$. Determining $u_{G}(p)$ is perhaps the most fundamental problem in the study of network reliability, with whole books on the topic [2? ]. Exact solution is intractable, as it was one of the original $\sharp P$ complete problems that Valiant [12] identified in his definition of the class $\sharp P$. However, a series of Fully Polynomial Approximation Schemes has been devised with ever-better runtimes [5-8], the previous best [8] being $O\left(n^{2.71}\right){ }^{1}$

We improve the time bound for approximating $u_{G}(p)$ to $\tilde{O}\left(n^{2}\right)$. We do so not with an entirely new algorithm, but with a deeper analysis of algorithms we developed in previous work. In particular, we also consider the partition function $z_{G}(p)$, the expected number of cuts that fail in $G$ when edges fail with probability $p$. We show that once $u_{G}(p)$ shrinks below $1 / 2$, the graph rapidly transitions to a phase where even $z_{G}(p)<1 / 2$, and in fact where $z_{G}(p)=$ $(1+o(1)) u_{G}(p)$. In other words, we are very unlikely to ever see more than one cut fail. This allows us essentially to treat these cut failures as independent, making it easier to estimate their likelihood. The contribution of this paper is not just the improved time bound, but also this clearer understanding of the evolution of a graph's graph's connectivity versus $p$.

To arrive at these results, we develop a new technique for assessing the structure of $G$ conditioned on a cut failure. This technique was pioneered by Harris and Srinivasan [5], but we simplify and strengthen it. We show that after the phase transition occurs, the failure of a cut does not substantially increase the likelihood of other cut failures; this near-independence in turn lets us prove strong bounds on the reliability behavior of $G$ in this regime. To show that the transition is rapid, we consider an evolutionary model of $G$ as edges are randomly contracted, and show how the small cuts rapidly disappear, leading to an accelerating rate of decrease in the size of the graph.

\subsection{Background}

In our previous work, we developed a natural unbiased estimator for $u_{G}(p)$-that is, an algorithm for generating a random variable whose expectation is $u_{G}(p)$. Given an unbiased estimator with relative variance $r$, a standard median-of-averages approach yields an $(\epsilon, \delta)$-approximation to $u_{G}(p)$-an approximation that is accurate to within $1 \pm \epsilon$ with probability $1-\delta$-that requires only $O\left(r \epsilon^{-2} \ln (1 / \delta)\right)=\tilde{O}(r)$ samples.

All previous work used Naive Monte Carlo (NMC) estimation to estimate sufficiently large $u_{G}(p)$. NMC simply generates $G(p)$-the result of deleting every edge independently with probability $p$-and returns 0 or 1 depending on whether the graph is connected or

\footnotetext{
${ }^{1}$ Recently, an FPRAS has also been developed [4] for the complementary problem of approximating the probability that the graph stays connected; however, neither approximation can be reduced to the other.
} 
disconnected. Since the probability of the measured event is $u_{G}(p)$, $\mathrm{NMC}$ is an unbiased estimator with relative variance $1 / u_{G}(p)$. Each sample can be generated easily in $O(m)$ time, or in $\tilde{O}(n)$ time using certain sparsification techniques [7], so the overall approximation runtime is $\tilde{O}\left(n / u_{G}(p)\right)$. In particular, when $u_{G}(p) \geq n^{-2}$ this runtime is $\tilde{O}\left(n^{3}\right)$.

For smaller $p$, we developed [7] a different unbiased estimator: generate a random sample $H$ by contracting each edge from $G$ with probability $1-q$ for some $q>p$-we write $H \sim G(q)$-then compute $u_{H}(p / q)$ recursively.

Lemma 1.1. For any $q$, the quantity $u_{H}(p / q)$ is an unbiased estimator for $u_{G}(p)$, meaning that $E\left[u_{H}(p / q)\right]=u_{G}(p)$.

Let $c$ be the minimum cut of $G$. Initially [7], we showed that when $p^{c} \leq n^{-2}$, we can set $q^{c}=n^{-2}$ and find that $u_{H}(p / q)$ has relative variance $n^{2}$. We also showed that $H$ has size $O(\log n)$ with high probability, so that the recursive computation of $u_{H}(p / q)$ is simple. Again using sparsification, we can generate each such $H$ in $\tilde{O}(n)$ time, which permits us to average the $\tilde{O}\left(n^{2}\right)$ samples needed for an FPRAS in $\tilde{O}\left(n^{3}\right)$ time. Combining with NMC for $p^{c} \geq n^{-2}$ gives us an $\tilde{O}\left(n^{3}\right)$ time algorithm over the full range of failure probabilities $p$.

In follow-on work [8] we improved our estimator using a strengthened relative variance bound. We proved the following lemma:

Lemma 1.2. Suppose that on every $n$-vertex graph $G$ with $u_{G}(p) \leq$ $n^{-\gamma}$, the relative variance of $u_{H}(p / q)$ is $\tilde{O}\left(q^{-c}\right)$. Then an unbiased estimator of constant relative variance for $u_{G}(p)$, and thus an $(\epsilon, \delta)$ approximation for $u_{G}(p)$, can be computed in $n^{\max (2,1+\gamma+o(1))}$ time.

Proof. We sketch the proof assuming the relative variance is $q^{-c}$ (ignoring the asymptotic factor). When $u_{G}(p) \geq n^{-\gamma}$ we can use NMC in $\tilde{O}\left(n^{1+\gamma}\right)$ time. Otherwise, we observed [8] that generating $H \sim G(q)$ and then computing an unbiased estimator for $u_{H}(p / q)$ recursively gave us an estimator for $G$ whose relative variance was the product of the two steps' variances. In particular, if we choose $q^{-c}=2$ then the relative variance of the recursive estimates gets doubled. Conversely, if we average two independent executions of this process, we halve the variance of the result, which cancels out that doubling and keeps the relative variance of the estimator small. We also know (from Corollary 2.2 below) that the size of the recursive subproblems is roughly $n / \sqrt{2}$. This yields a recursive algorithm with runtime $T(n)=2 T(n / \sqrt{2})+O\left(n^{2}\right)=\tilde{O}\left(n^{2}\right)$.

The algorithm of this proof is in effect the Recursive Contraction Algorithm (RCA) for minimum cuts [9]. Its $\tilde{O}\left(n^{2}\right)$ runtime improves on the previous $\tilde{O}\left(n^{3}\right)$ for $p^{c} \leq n^{-\gamma}$.

In the same work, we proved the hypothesis of the lemma with $\gamma=1.71$, which yielded an $\tilde{O}\left(n^{2.71}\right)$-time estimator algorithm. The bottleneck in the lemma is the need to apply NMC when $p^{c} \geq n^{-\gamma}$; the recursive algorithm for smaller $p$ runs in $\tilde{O}\left(n^{2}\right)$ time.

\subsection{Our Contribution}

In this work, we prove the hypothesis of Lemma 1.2 for every constant $\gamma>0$, thus improving the approximation time to $\tilde{O}\left(n^{2}\right)$. This result shows that we can use NMC so long as $u_{G}(p) \geq n^{-\epsilon}$, with runtime $\tilde{O}\left(n^{1+\epsilon}\right)$, and switch to the RCA, with runtime $\tilde{O}\left(n^{2}\right)$, for all smaller values.
For this analysis, we significantly strengthen the connection between reliability and estimator variance. We show that as the edge failure probability $p$ shrinks, any graph evolves through three distinct stages:

We define the breakpoint of $G$ to be the probability $b$ such that $u_{G}(b)=1 / 2$ (note $b$ must exist since $u_{G}(0)=0$ and $u_{G}(1)=1$ ). Above the breakpoint, NMC is fast. As $p$ moves below the breakpoint, $G$ is becoming unlikely to disconnect. However, it is possible for several cuts to fail simultaneously at this point, and the dependence between these failures makes the variance difficult to analyze.

Therefore, we identify a strongpoint $s \leq b$ where the probability of two distinct cut failures becomes negligible. Below this point, the different cut failures can be treated as independent, which makes analyzing them much simpler. In particular, we show that the relative variance of $u_{H}(p / q)$ becomes bounded by $q^{-c}$, so we can apply our recursive estimator.

This gives us a solution for $p \geq b$ (use NMC) and for $p<s$ (use the recursive estimator). It remains to handle the range between $b$ and $s$. We show that $s$ is very close to $b$, and that as a result $u_{G}(s)$ cannot decrease too much from $u_{G}(b)$, and in particular $u_{G}(s) \geq n^{-o(1)}$. This permits us to apply NMC all the way down to $s$, and then switch to the recursive estimator below $s$.

We have already mentioned the partition function

$$
z_{G}(p)=\sum_{i} p^{\left|C_{i}\right|}
$$

where the $C_{i}$ range over all cuts of $G$. Of particular importance in our new analysis is the pairability

$$
x_{G}(p)=\sum_{i \neq j} p^{\left|C_{i} \cup C_{j}\right|}
$$

This is the expected number of distinct pairs of cuts in $G(p)$, since $p^{\left|C_{i} \cup C_{j}\right|}$ is the probability that cuts $C_{i}$ and $C_{j}$ both fail. The pairability $x_{G}(p)$ appears in two key places: first, as the error term in the inclusion-exclusion expansion $u_{G}(p) \geq z_{G}(p)-x_{G}(p)$ that approximates $u_{G}(p)$ by the simpler $z_{G}(p)$, and second, through the pairwise terms that arise when we compute the variance $E\left[z_{H}(p / q)^{2}\right]$. We show that when $p \leq s$ we have $x_{G}(p) \ll z_{G}(p)$, which essentially allows us to treat all the cut failures as independent and upper bound the relative variance.

More precisely, we prove the following:

(1) The relative variance of $u_{H}(p / q)$ is at most $q^{-c}\left(1+\log _{p}(q)+\right.$ $\left.x_{G}(p) / z_{G}(p)\right)$ (Theorem 3.1).

(2) There is a strongpoint $s$ such that $x_{G}(p)=o\left(z_{G}(p)\right)$ for $p \leq s$ (Theorem 5.1).

(3) At the strongpoint $u_{G}(s) \geq n^{-o(1)}$ (Theorem 7.2).

Items 1 and 2 together show that for $p$ below the strongpoint, the relative variance is $q^{-c}(1+o(1))$ which means that the RCA can estimate the reliability in $\tilde{O}\left(n^{2}\right)$ time. Item 3 shows that for $p$ above the strongpoint, $u_{G}(p)$ is large, which means that NMC (with sparsification) can estimate the reliability in $\tilde{O}\left(n^{1+o(1)}\right)$ time. Combining these results yields our time bound.

To analyze the strongpoint, we develop an approach to estimating (other) cut failure events in $G$ conditioned on the failure of a particular cut. This approach was pioneered by Harris and Srinivasan [5], but we significantly simplify and strengthen it. 


\section{BACKGROUND}

\subsection{Unbiased Estimators}

We rely on unbiased estimators and their relative variance. We provide some background on these ideas in Appendix A.

For NMC, the unbiased estimator is the indicator variable for whether $G(p)$ is disconnected in an experiment; it has relative variance $1 / u_{G}(p)$. For our faster algorithms, we generate $H \sim G(q)$ and use $u_{H}(p / q)$ as our estimator $X$. Note that $H(p / q)$ has the same distribution as $G(p)$; thus $E\left[u_{H}(p / q)\right]=u_{G}(p)$.

In our previous work [8], we bounded the relative variance of $u_{H}(p / q)$ as an estimator for $u_{G}(p)$ by $q^{-c}$ when $p^{c} \leq n^{-2}$. Here we extend that result to any $p$ below the strongpoint $s$.

\subsection{Previous Reliability Bounds}

We make use of the following reliability bounds from previous work [7]:

Lemma 2.1. The size of $G(p)$ is stochastically dominated by a binomial distribution with parameters $n$ and $p^{c / 2}$.

(To be precise, $G(p)$ never has zero vertices while the binomial distribution can yield zero. However, defining the size of a onevertex graph to be 0 resolves the issue and creates no ambiguity.)

Corollary 2.2. The expected size of $G(p)$ is at most $n p^{c / 2}$.

Corollary 2.3. If $n^{2} p^{c} \leq 1$ then $z_{G}(p) \leq n^{2} p^{c} / 2$, and thus we always have $u_{G}(p) \leq n^{2} p^{c}$.

Corollary 2.4. $b^{c} \geq n^{-2} / 2$

\section{RELATIVE VARIANCE BY CUT PAIRS}

We begin by showing the key role that $x_{G}(p)$, the expected number of failed pairs of cuts in $G(p)$, plays in computing the relative variance. This analysis does not rely on any special properties of cuts in graphs; it applies to any monotone reliability system [1]: a collection of subsets of a ground set (e.g. cuts), where each element (e.g. edge) fails with probability $p$ and the system fails iff all elements of any subset do. For consistency, we continue to use the language of cuts.

Lemma 3.1. Suppose that $x_{G}(p)<z_{G}(p) / 2$. Then for $p \leq q$ the relative variance of $u_{H}(p / q)$ is

$$
\frac{E\left[u_{H}(p / q)^{2}\right]}{u_{G}(p)^{2}}=q^{-c}\left(1+O\left(\log _{p} q\right)+O\left(x_{G}(p) / z_{G}(p)\right)\right.
$$

When we apply the lemma we will have $q=\Omega(1)$ while $p=$ $n^{-\Omega(1)}$, so the second term will be $O(1 / \log n)$ and thus negligible. This section is devoted to proving this lemma.

For comparison, suppose $G$ has two vertices with $c$ connecting edges. Then $H$ is $G$ and has $u_{H}(p / q)=(p / q)^{c}$ with probability $q^{c}$, and a single vertex with $u_{H}(p / q)=0$ otherwise. Thus, the relative variance is $q^{c}(p / q)^{2 c} / p^{2 c}=q^{-c}$. Thus, our bound is tight aside from the error terms.

\subsection{Variance of a Sum}

It's well known that summing identical independent random variables decreases the relative variance. It becomes more complicated when the variances being summed are not identical. We show that in this case at least the relative variance does not get (much) worse.

Suppose that we were to compute the relative variance of $z_{H}(p / q)$ under the assumption that each cut fails independently of the others so the variance is the sum of individual cut variances. Then cut $C_{i}$ of value $c_{i}$ survives in $H$ with probability $q^{c_{i}}$ and contributes a value of $(p / q)^{c_{i}}$ to $z_{H}(p / q)$ if so; it follows that its contribution to the variance is $q^{c_{i}}(p / q)^{2 c_{i}}=\left(p^{2} / q\right)^{c_{i}}$. Thus the overall relative variance is $\sum\left(p^{2} / q\right)^{c_{i}} /\left(\sum p^{c_{i}}\right)^{2}$. The largest single contribution of $\left(p^{2} / q\right)^{c}$ comes from the minimum cut, and if this is the only cut then the relative variance is $q^{-c}$. Adding other, independent cuts should only decrease that relative variance; we show this is at least approximately true.

Lemma 3.2. Let $p \leq q$ and all $c_{i} \geq c$. Then

$$
\frac{\sum\left(\left(p^{2} / q\right)^{c_{i}}-p^{2 c_{i}} / q^{c}\right)}{\left(\sum p^{c_{i}}\right)^{2}}=O\left(q^{-c} \log _{p} q\right)
$$

In particular, the quantity is $O\left(q^{-c} / \log n\right)$ in our use case where $q^{c}=1 / 2$ and $p^{c} \leq 1 / n$. That is, compared to the "hoped for" relative variance $q^{-c}$, the overall difference is negligible.

The two parts in each term of the numerator are quite similar. For minimum cuts the difference is 0 . For larger cuts, there is a growing difference $q^{-c_{i}}-q^{-c}$, but this difference is damped by a multiplicative factor of $p^{2 c_{i}}$ making it contribute less to the sum. Thus, small cuts (and their small-ratio differences) dominate that sum and the overall ratio.

Because the proof simply applies standard calculus, we place it in Appendix B.

\subsection{The Relative Variance}

We now prove Lemma 3.1. From the inclusion-exclusion formula, we know that $z_{G}(p)-x_{G}(p) \leq u_{G}(p) \leq z_{G}(p)$. We can therefore bound

$$
\begin{aligned}
\frac{E\left[u_{H}(p / q)^{2}\right]}{u_{G}(p)^{2}} & \leq \frac{E\left[z_{H}(p / q)^{2}\right]}{\left(z_{G}(p)-x_{G}(p)\right)^{2}} \\
& =\frac{E\left[z_{H}(p / q)^{2}\right]}{z_{G}(p)^{2}\left(1-x_{G}(p) / z_{G}(p)\right)^{2}} \\
& =\frac{E\left[z_{H}(p / q)^{2}\right]}{z_{G}(p)^{2}}\left(1+O\left(x_{G}(p) / z_{G}(p)\right)\right)
\end{aligned}
$$

when $x_{G}(p)<z_{G}(p) / 2$.

Thus, we now focus on bounding the relative variance of $z_{H}(p / q)$ that appears here before the error term. First consider the numerator. For cuts $C_{i}$ and $C_{j}$ write $p^{C_{i} \cup C_{j}}$ as shorthand for $p^{\left|C_{i} \cup C_{j}\right|}$, write $p^{C_{i}+C_{j}}$ for $p^{\left|C_{i}\right|+\left|C_{j}\right|}$, and write $p^{C_{i} \cap C_{j}}$ for $p^{\left|C_{i} \cap C_{j}\right|}$. Also write $S_{i}$ for the event that cut $C_{i}$ is present in $H$, which happens with probability $q^{c_{i}}$. Then

$$
\begin{aligned}
E\left[z_{H}(p / q)^{2}\right] & =E\left[\left(\sum S_{i} \cdot(p / q)^{C_{i}}\right)^{2}\right] \\
& =\sum_{i, j} E\left[S_{i} S_{j} \cdot(p / q)^{C_{i}+C_{j}}\right] \\
& =\sum q^{C_{i} \cup C_{j}}(p / q)^{C_{i}+C_{j}}
\end{aligned}
$$


We bound this sum in three parts. First, consider pairs with $i=j$, for which

$$
\sum_{i} q^{C_{i} \cup C_{j}}(p / q)^{C_{i}+C_{j}}=\sum_{i} p^{2 c_{i}} / q^{c_{i}}
$$

Second, consider those pairs $i \neq j$ for which $\left|C_{i} \cap C_{j}\right| \geq c$. Then

$$
\begin{aligned}
q^{C_{i} \cup C_{j}}(p / q)^{C_{i}+C_{j}} & =(p / q)^{C_{i} \cap C_{j}} p^{C_{i} \cup C_{j}} \\
& \leq(p / q)^{c} p^{C_{i} \cup C_{j}}
\end{aligned}
$$

since $p \leq q$. So the sum over all such pairs $i \neq j$ is bounded by

$$
(p / q)^{c} \sum_{i \neq j} p^{C_{i} \cup C_{j}}=(p / q)^{c} x_{G}(p)
$$

Note that we expanded the sum to include all pairs $i \neq j$, but this only raises our upper bound. Third, consider those $i \neq j$ for which $\left|C_{i} \cap C_{j}\right| \leq c$. Then

$$
\begin{aligned}
q^{C_{i} \cup C_{j}}(p / q)^{C_{i}+C_{j}} & =p^{C_{i}+C_{j}} / q^{C_{i} \cap C_{j}} \\
& \leq p^{C_{i}+C_{j}} / q^{c} .
\end{aligned}
$$

It follows that the sum over all such pairs is at most

$$
q^{-c} \sum_{i \neq j} p^{c_{i}+c_{j}}=q^{-c}\left(z_{G}(p)^{2}-\sum_{i} p^{2 c_{i}}\right) .
$$

Again, we expanded to all pairs $i \neq j$ which only increases the bound.

Putting these parts together, we find that

$$
\begin{aligned}
\frac{E\left[z_{H}(p / q)^{2}\right]}{z_{G}(p)^{2}} & \leq \frac{\sum_{i}\left(p^{2} / q\right)^{c_{i}}+q^{-c}\left(z_{G}(p)^{2}-\sum_{i} p^{2 c_{i}}\right)+(p / q)^{c} x_{G}(p)}{z_{G}(p)^{2}} \\
& =\frac{\sum_{i}\left(\left(p^{2} / q\right)^{c_{i}}-p^{2 c_{i}} / q^{c}\right)+q^{-c} z_{G}(p)^{2}+(p / q)^{c} x_{G}(p)}{z_{G}(p)^{2}} \\
& =\frac{\sum_{i}\left(p^{2} / q\right)^{c_{i}}-p^{2 c_{i}} / q^{c}}{z_{G}(p)^{2}}+q^{-c}+\frac{(p / q)^{c} x_{G}(p)}{z_{G}(p)^{2}} \\
& \leq q^{-c} \log _{p}(q)+q^{-c}+q^{-c} x_{G}(p) / z_{G}(p)
\end{aligned}
$$

where for the first term we apply Lemma 3.2 and for the last we note that $z_{G}(p) \geq p^{c}$,

$$
=q^{-c}\left(1+\log _{p}(q)+x_{G}(p) / z_{G}(p)\right)
$$

This bounds the relative variance of $z_{H}(p / q)$; to get the bound for $u_{H}(p / q)$ we must multiply this quantity by the outer error term $\left(1+O\left(x_{G}(p) / z_{G}(p)\right)^{2}\right)$, but this adds only a smaller error (under the assumption of the lemma that $\left.x_{G}(p) / z_{G}(p)<1 / 2\right)$ that is absorbed in the $O\left(x_{G}(p) / z_{G}(p)\right)$ term that is already present.

\section{CUT COUNTING BOUNDS}

In this section, we give a variety of bounds on the number of cuts in a graph subject to certain conditions, and more generally on several variants of the partition function $z_{G}(p)$, that are needed in our analysis. Our approach is to apply the Contraction Algorithm-a stochastic process that selects a graph cut by randomly contracting edges until only one cut remains. By modifying the stochastic process, we arrange for the selection probability of any cut to upper bound that cut's contribution to the variant partition function.
Then, recognizing that only one cut is selected, so that the expected number of selected cuts is 1 , we conclude that the sum of these upper bounds is at most 1, yielding an upper bound on the variant partition function.

\subsection{A Product Lemma}

Our analysis requires the following product approximation, which we proved previously [8]. It is related to the well known bound $1+x \leq e^{x}$.

Lemma 4.1 (Product Approximation). Consider a product $P=$ $\prod_{r \geq 2 \gamma}\left(1-x_{r}\right)$ such that $x_{r} \leq \gamma / r$ and let $S=\sum_{r \geq 2 \gamma} x_{r}$. Then

$$
2^{-\gamma} \exp (-S) \leq P \leq \exp (-S)
$$

\subsection{The Contraction Algorithm}

The Contraction Algorithm repeatedly chooses and contracts a random edge of $G$ until some stopping condition is reached; we say that a cut survives the run of the Contraction Algorithm if no edge of the cut is ever chosen. We define an $\alpha$-min-cut to be one of value at most $\alpha$ times the minimum.

Lemma 4.2 ([9]). Any $\alpha$-minimum cut survives contraction to $4 \alpha$ vertices with probability at least $(\alpha / n)^{2 \alpha}$.

Proof. When $r$ vertices remain, a graph with min-cut $c$ has at least $r c / 2$ edges. Thus, the probability that a randomly chosen edge is in the cut under consideration is at most $2 \alpha / r$. Thus, the probability that such an edge is never chosen is $\prod(1-2 \alpha / r)$. Now apply Lemma 4.1 with $\gamma=2 \alpha$ and $\sum_{r=4 \alpha}^{n} 2 \alpha / n \leq 2 \alpha \ln (n / 4 \alpha)$.

\subsection{Conditioning on Cut Failures}

Our analysis below assesses the outcome of $G(q)$ conditioned on the failure of a particular cut $A$ in $G(q)$. We can generate such conditional samples in a straightforward way: we delete all edges of $G$ with probability $q$ (equivalently, we can contract those that remain, each with probability $1-q$ ), but then also delete all edges of $A$ so that $A$ is a cut in the sample.

Lemma 4.3. Suppose that $A$ is an $\alpha$-minimum cut of $G$ and $p$ is such that $u_{G}(p) \leq 1 / 2\left(\right.$ resp. $\left.z_{G}(p) \leq 1\right)$. Then conditioned on $A$ surviving, the graph $G(p)$ has $O(\alpha \log n)$ components (which become vertices upon contraction) with probability at least $1 / 4$ (resp. with high probability).

Proof. If $u_{G}(p)=1 / 2$ then by definition $G(p)$ is connected with probability at least $1 / 2$. Alternatively suppose $z_{G}(p)=1$. By definition $z_{G}(p)$ is the expected number of cuts that survive in $G(p)$. It follows from Markov's inequality that $G(p)$ has $n^{O(1)}$ surviving cuts with high probability. However, if $G(p)$ has $k$ components, then it has $2^{k-1}$ surviving cuts. Thus, the number of components $k=O(\log n)$ with high probability.

Now suppose that we condition on the survival of cut $A$ in $G(p)$. We can do so by first generating $G(p)$ and then deleting any edges of $A$ that are included. Deleting these edges increases the number of components of $G(p)$ by at most the number of edges deleted.

If $u_{G}(p)=1 / 2\left(\right.$ resp. $\left.z_{G}(p)=1\right)$ then Corollary 2.3 implies that $p^{c} \geq n^{-2}$. It follows that every edge is sampled in $G(p)$ with probability $1-p \leq 1-n^{-2 / c}=1-e^{-2(\ln n) / c}=O((\log n) / c)$. 
From a Chernoff bound, we conclude that with high probability $O(\alpha \log n)$ edges of $A$ are sampled. Deleting these sampled edges can only increase the number of components of $G(p)$ by $O(\alpha \log n)$.

If $G(p)$ has few vertices and few edges of $A$ are sampled, then the total number of components is $O(\log n)+O(\alpha \log n)=O(\alpha \log n)$. By a union bound on the complementary events, this happens with probability at least $1 / 4$ when $u_{G}(p)=1 / 2$ and with high probability when $z_{G}(p)=1$.

For the more general case $u_{G}(p) \leq 1 / 2\left(\right.$ resp. $\left.z_{G}(p) \leq 1\right)$, we need consider only smaller $p^{c} \leq n^{-2}$ which only increases the probability that edges of $G-A$ are included; thus it cannot increase the number of components that result.

Remark. Our analysis for the $z_{G}(p)$ case is similar to the standard Chernoff Bound. The Chernoff analysis applies Markov's inequality to the exponential generating function $E\left[e^{t X}\right]$. We do the same, because the exponential generating function for the number of vertices $h$ in $H$ is simply $E\left[2^{h}\right]=2 z_{G}(p)$.

Recall that we defined the breakpoint $b$ of $G$ such that $u_{G}(b)=$ $1 / 2$. For $p \geq b$ the graph $G$ is unreliable $\left(u_{G}(p)>1 / 2\right)$. We show that for $p$ just slightly less than $b$, the unreliability structure of the graph becomes quite simple.

Lemma 4.4. There is an such that $s^{c}=\Omega\left(b^{c} / \ln ^{2} n\right)$ and $z_{G}(s) \leq$ $1 / 2$.

This says that at a $p$ just slightly smaller than where $G(p)$ becomes reliable, its expected number of failed cuts becomes small. This result is a special case of the recent breakthrough result on the fractional expectation threshold [3], but we give a simpler direct proof specialized to cuts.

Proof. Consider the following procedure for choosing a cut from $G$. Choose a target graph size $k \geq 2$ with probability $2^{1-k}$ (so these probabilities sum to 1 ). Then, choose $H \sim G(b)$ and contract its components. If the result has more than $k$ vertices, then run the Contraction Algorithm on $H$ until $k$ vertices remain. Finally, choose at random from among the at most $2^{k-1}$ cuts of the resulting graph.

What is the probability that a given $\alpha$-minimum cut $A$ is chosen? At a minimum, it is chosen if (i) none of its edges are contracted in creating $H$, (ii) we choose $k=4 \alpha$, (iii) the cut $A$ survives contraction to $k=4 \alpha$ vertices, and (iv) the cut is chosen from the $2^{k-1}$ possibilities. Events (ii) and (iv) each happen with probability $2^{1-4 \alpha}$. Event (i) happens with probability $b^{\alpha c}$.

The only complicated event is (iii). If graph $H$ has $h$ vertices, Lemma 4.2 shows that $A$ survives the contractions with probability at least $(\alpha / h)^{2 \alpha}$. By Lemma 4.3, $h=O(\alpha \log n)$ with constant probability, in which case $(\alpha / h)^{2 \alpha}=(\alpha / O(\alpha \ln n))^{2 \alpha}=\Omega(1 / \ln n)^{2 \alpha}$.

We now multiply the probabilities of our four events to conclude that $A$ is chosen with probability at least $4^{1-4 \alpha} b^{\alpha c} \Omega(1 / \ln n)^{2 \alpha}=$ $\Omega\left(b^{c} / \ln ^{2} n\right)^{\alpha}$. Since only one cut is chosen, summing these lower bounds must yield a value less than 1 . That is, indexing over all cuts $i$ with respective sizes $\alpha_{i} c$,

$$
\sum_{i} \Omega\left(b^{c} / \ln ^{2} n\right)^{\alpha_{i}} \leq 1
$$

Note that the exponent $\alpha_{i}$ is intentionally outside the asymptotic $\Omega($ ), to make the claim valid for non-constant $\alpha$ as well.
If we now determine $s$ by setting $s^{c}$ equal to the $\Omega\left(b^{c} / \ln ^{2} n\right)$ term in the formula just above, we conclude that $z_{G}(s)=\sum s^{\alpha_{i} c} \leq 1$. If we divide this $s$ by $2^{1 / c}$, dividing $s^{c}$ by 2 (so we still have $s^{c}=$ $\left.\Omega\left(b^{c} / \ln ^{2} n\right)\right)$, then since each $\alpha_{i} \geq 1$, we divide each term in $z_{G}(s)$ by at least 2 and ensure $z_{G}(s) \leq 1 / 2$. This completes our proof. $\quad \square$

Remark. We performed this analysis using a sampling step followed by a contraction step, but in fact we have also proven a new property of the Contraction Algorithm [9]. Our first sampling step can be seen as executing a large number of contractions in parallel instead of sequentially. More precisely, recall that we can implement the Contraction Algorithm by randomly permuting the edges, then contracting in permutation order until the requisite size is reached. Here is an indirect way to do that. First, sample a binomial distribution $B(m, s)$ and place that many random edges at the tail of the permutation and the remaining edges at the head of the permutation. Now randomly permute the head and tail edges to get the entire permutation. In the event that the edges in the head of the permutation fail to contract the graph to $k$ vertices, the permutation of edges in the tail will determine the outcome. But the edges in the tail are precisely the edges of $H$. Our analysis above therefore proves that with constant probability the tail permutation will determine the outcome, demonstrating:

Corollary 4.5. In a graph with breakpoint b, the Contraction Algorithm outputs each $\alpha$-min-cut with probability $\Omega\left(b^{c} / \ln n\right)^{\alpha}$.

In other words, the Contraction Algorithm for finding min-cuts is less error prone, and can thus be faster, on reliable graphs (which have large breakpoints). Observe that the Contraction Algorithm does not need to know $b$ for this result to hold. But to find the min-cut with high probability, we can use $O(\log n)$ iterations of NMC, taking $\tilde{O}(m)$ time, to determine $b$ and use it to adjust the number of Contraction Algorithm iterations needed for success on a given graph, making the Contraction Algorithm "self tuning."

\subsection{Interacting Cuts}

We now prove some bounds on the probability that a given cut survives in $G(p)$ conditioned on the survival of some other cut. We first prove a universal bound that is true of all graphs; we then give a tighter bound parametrized by the breakpoint $b$.

\subsubsection{A Universal Bound.}

Lemma 4.6. Fix any $\alpha$-minimum cut $A$ of $G$, and for every other cut $B_{j}$ let $\beta_{j} c$ be the number of edges of $B_{j}$ not in $A$. Then

$$
\sum_{j} 16^{-\alpha}\left(\beta_{j} / 8 n\right)^{2 \beta_{j}} \leq 1
$$

Proof. Consider a cut $A$ with $\alpha c$ edges and a cut $B$ that has $\beta c$ edges in $G-A$. Suppose we run the Contraction Algorithm on the graph $G-A$ until the graph has $2 \alpha+4 \beta$ vertices. When $s$ vertices remain the graph $G$ has $s c / 2$ edges so $G-A$ has at least $s c / 2-\alpha c$ edges. This makes the probability that we choose an edge of $B$ at most $2 \beta /(s-2 \alpha)$. Thus, the probability that no edge of $B$ is chosen 
in any step is at least (taking $\gamma=2 \beta$ and $r=s-2 \alpha$ in Lemma 4.1)

$$
\begin{aligned}
\prod_{s=2 \alpha+4 \beta}^{n}\left(1-\frac{2 \beta}{s-2 \alpha}\right) & \geq 2^{-2 \beta} \exp \left(-2 \beta \sum_{s=2 \alpha+4 \beta}^{n} \frac{1}{s-2 \alpha}\right) \\
& \geq 2^{-2 \beta} \exp (-2 \beta(\ln n-\ln 4 \beta)) \\
& =2^{-2 \beta}(4 \beta / n)^{2 \beta} \\
& =(2 \beta / n)^{2 \beta}
\end{aligned}
$$

We can use this as we did before in a general procedure for choosing a cut in $G-A$. First, set a target graph size $k>2 \alpha$ with probability $2^{2 \alpha-k}$ (so the probabilities sum to 1 ). Then, contract $G-A$ until it has $k$ vertices. Finally, choose at random from among the $2^{2 \alpha-k}$ cuts of this graph. The probability that we choose cut $B$ with its $\beta c$ edges not in $G-A$ is at least the probability that we choose $k=\lceil 2 \alpha+4 \beta\rceil$, times the probability that $B$ survives contraction to $k$ vertices, which we showed above to be at least $(2 \beta / n)^{2 \beta}$, times the probability that we choose $B$ in the resulting graph, which is at least $2^{-2 \alpha-4 \beta}$. Multiplying these terms gives us an overall bound of

$$
\begin{aligned}
4^{-2 \alpha-4 \beta}(2 \beta / n)^{2 \beta} & =16^{-\alpha-2 \beta}(2 \beta / n)^{2 \beta} \\
& =16^{-\alpha}(\beta / 8 n)^{2 \beta}
\end{aligned}
$$

Since only one cut is output, it follows that the sum of these probability lower bounds, which lower bounds the expected number output, is at most 1 .

4.4.2 A Parametrized Bound. We can derive a more precise bound based on the breakpoint parameter $b$, applying the same construction as we did in Section 4.3 for single cuts.

Lemma 4.7. Fix any $\alpha$-minimum cut $A$ of $G$, and for every other cut $B_{j}$ let $\beta_{j} c$ be the number of edges of $B_{j}$ not in $A$. Then

$$
\sum_{\beta_{j} \geq \alpha / 2} \Omega\left(b^{c} / \ln ^{2} n\right)^{\beta_{j}} \leq 1
$$

Proof. Consider the following procedure for choosing a cut of $G-A$. First, generate $H \sim(G-A)(b)$. Then, use the Contraction Algorithm on $H-A$, as for the unparametrized bound of Lemma 4.6, to generate a cut of $H$ distinct from $A$. The analysis proceeds exactly as in Section 4.4.1. We choose cut $B$ with $\beta c$ edges in $G-A$ if first $B$ survives in the graph $H-A$ and then $B$ is the cut chosen by the contraction algorithm on $H-A$. The probability of the first event is $b^{\beta c}$. It remains to bound the probability that $B$ is chosen by contraction of $H$, conditioned on its surviving in $H$. To determine this probability, we apply the previous universal bound that depends only on the size of $H-A$. Thus, we need to bound the size of $H-A$. However, we also need to condition on having chosen no edges of $B-A$. Putting these together, it follows that we must bound the size of $(G-A-B)(b)$. Arguing exactly as in Lemma 4.3, we can conclude that with probability at least $1 / 4, H \sim(G-A-B)(b)$ will have at most $8(\alpha+\beta) \ln n$ vertices.

If this happens, then we can substitute $8(\alpha+\beta) \ln n$ for $n$ in the universal bound of Lemma 4.6 and conclude that $B$ will then be chosen by the contraction algorithm in $H-A$ with probability at least

$$
16^{-\alpha}(\beta / 8(\alpha+\beta) \ln n)^{2 \beta}
$$

Our sum considers only cuts for which $\beta \geq \alpha / 2$, in which case we can lower bound the above by

$$
16^{-2 \beta}\left(\frac{\beta}{8(3 \beta) \ln ^{2} n}\right)^{\beta}=\Omega(\ln n)^{-2 \beta} .
$$

We now multiply by the probability $b^{\beta c}$ that $B$ survives in $H-A$, and conclude that the probability cut $B$ is chosen by the overall procedure is $\Omega\left(b^{c} / \ln ^{2} n\right)^{\beta}$. Since we choose only one cut, this implies that if we consider all cuts $B_{j}$ with $\beta_{j} c$ edges not in $A$, the expected number chosen is

$$
\sum_{\beta_{j} \geq \alpha / 2} \Omega\left(b^{c} / \ln ^{2} n\right)^{\beta_{j}} \leq 1 .
$$

Corollary 4.8. There is an $s$ with $s^{c}=\Omega\left(b^{c} / \log ^{2} n\right)$ such that, conditioned on the failure of $\alpha$-minimum cut $A$, the probability that some other cut of $G$ fails that has more than $\alpha / 2$ edges not in $A$ is at most $1 / 2$.

Proof. If we set $s^{c}=\Omega\left(b^{c} / \log ^{2} n\right)$ per the previous lemma, then $\sum s^{\beta c} \leq 1$. But this is the expected number of cuts that fail that satisfy the condition of the corollary. If we decrease $s$ by $2^{1 / c}$ then $s^{c}$ is unchanged asymptotically, but now $\sum s^{\beta c} \leq 1 / 2$ as required. This is the expected number of failures; now apply the union bound.

\section{THE RELATIVE VARIANCE}

The bound of Section 3 applies to any monotone reliability system. We now use the special properties of cuts derived in Section 4 to develop our bound on the pairability $x_{G}(p)$.

Theorem 5.1. There is a strongpoint $s$ such that $s^{c}=\Omega\left(b^{c} / \log ^{2} n\right)$ and $x_{G}(p) \leq(p / s)^{c / 2} z_{G}(p)$ for $p \leq s$.

Corollary 5.2. For $p \leq s$ the relative variance of $u_{H}(p / q)$ is

$$
q^{-c} \cdot O\left(1+\log _{p} q+(p / s)^{c / 2}\right)
$$

In particular, when $p^{c} \leq \min \left(n^{-.01}, s^{c} / \log n\right)$ and $q^{-c}=\Theta(1)$, this is $q^{-c}(1+O(1 / \log n))$.

To prove the theorem, we use the same approach as in our previous work [7], but apply our new conditional cut failure bounds. The corollary follows immediately from Theorem 3.1.

We rely on a lemma from our previous work. It shows that as we sum over cut pairs, we can assume the intersection of each pair we are considering is substantial.

Lemma 5.3 ([8]). For each cut $i$ we can define a set of associated cuts $A_{i}$ such that every $C_{j} \in A_{i}$ satisfies $\left|C_{j}-C_{i}\right| \geq \frac{1}{2}\left|C_{i}\right|$ and

$$
x_{G}(p)=\sum p^{C_{i} \cup C_{j}}=\frac{3}{2} \sum_{i} p^{C_{i}} \sum_{j \in A_{i}} p^{C_{j}-C i} .
$$

Proof. (Sketch) For intuition, consider the three cuts of a triangle on 3 vertices-each cut consists of two edges, and each edge is the intersection of exactly two of the three cuts. We showed [8] that this holds in general: for any graph, each pair of cuts $C_{i}$ and $C_{j}$ defines an XOR-cut $C_{k}$ with the following characteristics. Each edge crossing any of the cuts crosses exactly two of them which means that each cut is a union of two disjoint semicuts, $S_{i}=C_{j} \cap C_{k}$, 
$S_{j}=C_{i} \cap C_{k}$, and $S_{k}=C_{i} \cap C_{j}$. Conversely, $C_{i}=S_{j} \cup S_{k}, C_{j}=S_{i} \cup S_{k}$, and $C_{k}=S_{i} \cup S_{j}$. All three pairs of cuts have the same union: $C_{i} \cup C_{j}=C_{j} \cup C_{k}=C_{k} \cup C_{i}=S_{i} \cup S_{j} \cup S_{k}$.

It follows that the cut-pair terms that appear in $x_{G}(p)$ can be grouped into sets of three, each consisting of the three pairs from a particular trio of cuts in the relationship just described, that is $p^{C_{i} \cup C_{j}}+p^{C_{j} \cup C_{k}}+p^{C_{i} \cup C_{k}}$ where $C_{k}$ is the XOR-cut of $C_{i}$ and $C_{j}$ (and vice versa). Without loss of generality, index the three cuts in size order $\left|C_{i}\right| \leq\left|C_{j}\right| \leq\left|C_{k}\right|$, which means that $\left|S_{i}\right| \geq\left|S_{j}\right| \geq\left|S_{k}\right|$. Thus $\left|C_{j}-C_{i}\right|=\left|S_{i}\right| \geq \frac{1}{2}\left(\left|S_{j} \cup S_{k}\right|\right)=\frac{1}{2}\left|C_{i}\right|$, so we can place $C_{j}$ (and similarly $C_{k}$ ) in $A_{i}$.

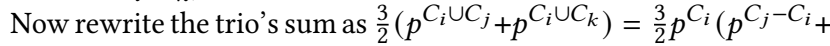
$\left.p^{C_{k}-C_{i}}\right)$. Note that we have dropped one of the three (equivalent) pairs; thus the scaling up by $3 / 2$ to compensate. Doing the same rewrite for each trio allows us to sum only over the sets $A_{i}$, so our sum can be rewritten as $\frac{3}{2} \sum_{i} p^{C_{i}} \sum_{j \in A_{i}} p^{C_{j}-C_{i}}$ as claimed.

We now prove Theorem 5.1. Take the $A_{i}$ of Lemma 5.3. If $C_{i}$ is an $\alpha$-minimum cut and we write $\left|C_{j}-C_{i}\right|=\beta_{j} c$, we know $\beta_{j} \geq \alpha / 2$ for all $C_{j} \in A_{i}$. Thus Lemma 4.7 applies and bounds $\sum_{j \in A_{i}} \Omega\left(b^{c} / \ln ^{2} n\right)^{\beta_{j}} \leq 1$. It follows that there is some $s$ with $s^{c}=$ $\Omega\left(b^{c} /(\ln n)^{2}\right)$ such that $\sum_{j \in A_{i}} s^{\beta_{j} c} \leq 1 / 3$. Thus for any $p \leq s$ we have $p^{\beta_{j} c}=(p / s)^{\beta_{j} c} s^{\beta_{j} c} \leq(p / s)^{\alpha_{i} c / 2} s^{\beta_{j} c} \leq(p / s)^{c / 2} s^{\beta_{j} c}$, so $\sum_{j \in A_{i}} p^{\beta_{j} c} \leq(p / s)^{c / 2} \sum_{j \in A_{i}} s^{\beta_{j} c} \leq \frac{1}{3}(p / s)^{c / 2}$, implying that

$$
\begin{aligned}
x_{G}(p)=\sum p^{C_{i} \cup C_{j}} & \leq \frac{3}{2} \sum_{i} p^{C_{i}} \sum_{j \in A_{i}} p^{C_{j}-C_{i}} \\
& \leq \frac{3}{2} \sum_{i} p^{C_{i}} \frac{1}{3}(p / s)^{c / 2} \\
& =\frac{1}{2}(p / s)^{c / 2} z_{G}(p)
\end{aligned}
$$

\section{THE FAILURE PHASE TRANSITION}

We have now completed our analysis of two of the three interesting ranges for $p$. When $p \geq b$ we can use NMC in a fast estimator. We have also just shown in Corollary 5.2 that when $p$ is less than the strongpoint $s$, the relative variance is $O\left(q^{-c}\right)$ which permits us to use the Recursive Contraction Algorithm [8] in an $\tilde{O}\left(n^{2}\right)$-time estimator.

It remains to consider the narrow range $b \geq p \geq s$ (recall that $s^{c}=\Omega\left(b^{c} / \log ^{2} n\right)$. We now prove that because $s$ is so close to $b$, the fact that $u_{G}(b)=1 / 2$ forces $u_{G}(s)$ to be large as well:

Lemma 6.1. $u_{G}(s)=n^{-1 / 2-o(1)}$.

In Section 7 we give a much stronger bound. But even this lemma shows that the runtime of NMC will be $n^{3 / 2+o(1)}=O\left(n^{2}\right)$ all the way down to the strongpoint $s$ where we can switch to the RCA.

Intuitively, this lemma makes sense. We showed in Theorem 5.1 that $u_{G}(s) \geq z_{G}(s)-x_{G}(s) \approx z_{G}(s)$. The function $z_{G}(p)$ has leading terms of the form $p^{-c}$, and as we decrease $p$ from $b$ to $s$, such terms decrease by a factor of only $O\left(\log ^{2} n\right)$. Since $z_{G}(b) \geq$ $u_{G}(b)=1 / 2$, this would suggest that $u_{G}(s) \approx z_{G}(s)=\Omega\left(1 / \log ^{2} n\right)$. However, most of the terms in $z_{G}(p)$ are associated with much larger cuts, so decrease much more rapidly as we decrease $p$ from $b$ to $s$. Conceivably, the large value of $z_{G}(b) \geq 1 / 2$ is generated entirely by large cuts, whose contribution vanishes away entirely by the time $p$ shrinks to $s$.

We demonstrate that this cannot happen, proving that a substantial portion of $z_{G}(b)$ is generated by small cuts, which therefore also contribute a substantial quantity to $u_{G}(s) \approx z_{G}(s)$. To do so, define $u_{G, \alpha}(p)$ to be the probability that some $\alpha$-minimum cut fails in $G(p)$, and similarly define $z_{G, \alpha}(p)$ to be the expected number of such cuts.

Lemma 6.2. $u_{G}(s)=\Omega\left(z_{G, \alpha}(b) / \log ^{2 \alpha} n\right)$

That is, if $z_{G, \alpha}(b)$ is large for any constant $\alpha$, then so is $u_{G}(s)$.

Proof. Consider a cut of value $c_{i} \leq \alpha c$ contributing to $z_{G, \alpha}(p)$. The ratio of the terms corresponding to this cut in $z_{G, \alpha(b)}$ and $z_{G, \alpha}(s)$ is $(s / b)^{c_{i}} \geq(s / b)^{\alpha c}=\left((s / b)^{c}\right)^{\alpha}=\Omega\left(\log ^{-2} n\right)^{\alpha}$ by Theorem 5.1, which proves $z_{G, \alpha}(s)=\Omega\left(z_{G, \alpha}(b) / \log ^{2 \alpha} n\right)$. Now note $z_{G}(s) \geq z_{G, \alpha}(s)$ and $u_{G}(s)=\Omega\left(z_{G}(s)\right)$.

Thus, for the remainder of this section we focus on lower bounding $z_{G, \alpha}(b)$.

\subsection{The Basic Idea}

We begin by proving $z_{G, \alpha}(b) \geq n^{-1-O(1 / \alpha)}$ which implies that $u_{G}(s) \geq n^{-1-o(1)}$. We reuse the trick of generating $G(b)$ in two steps, first generating $H \sim G(q)$ and then generating $G(b)$ as $H(b / q)$, but draw a new conclusion: if $z_{G, \alpha}(b)$ is small, then $H$ is unlikely to have any surviving small cuts. And if $H$ has no small cuts then $H(b / q)$ is very likely to be connected, which means that $u_{G}(b)=E\left[u_{H}(b / q)\right]$ is close to 1 . But this cannot happen, because $b$ was defined so that $u_{G}(b)=1 / 2$.

Write $z=z_{G, \alpha}(b)$. We assume $z \leq 1 / 4$ (since otherwise we are done) and define $q=b /(4 z)^{1 / \alpha c} \geq b$. Define $H \sim G(q)$ so $G(b) \sim H(b / q)$.

Since the degree of polynomial $z_{G, \alpha}(p)$ is bounded by $\alpha c$, we know $z_{G, \alpha}(q) \leq(q / b)^{\alpha c} z_{G, \alpha}(b)=z / 4 z=1 / 4$. It follows by Markov's inequality that $H$ has a cut of value less than $\alpha c$ with probability at most $1 / 4$. Furthermore, Corollary 2.2 states that $H$ has size $O\left(n^{2} q^{c / 2}\right)$ w.h.p; in particular there is some $h=O\left(n^{2} q^{c / 2}\right)$ such that the probability $H$ has size greater than $H$ is at most $1 / 8$. Thus, the probability of either "bad" outcome is at most $3 / 8$.

If neither bad outcome happens then $H$ has size $h$ and min-cut at least $\alpha c$, so $u_{H}(b / q) \leq h^{2}(b / q)^{\alpha c}=4 z h^{2}$ by Corollary 2.3. Since $E\left[u_{H}(b / q)\right]=u_{G}(b)=1 / 2$, and since since the bad outcomes occur with total probability at most $3 / 8$, this last case must contribute at least $1 / 8$ to the disconnection probability. That is, $4 z h^{2} \geq 1 / 8$ meaning $z \geq 1 / 32 h^{2}$. But $h^{2}=O\left(n^{2} q^{c}\right)=O\left(n^{2} b^{c} /(4 z)^{1 / \alpha}\right)$, which lets us conclude that

$$
\begin{aligned}
z & =\Omega\left(z^{1 / \alpha} n^{-2} b^{-c}\right) \\
z^{1-1 / \alpha} & =\Omega\left(n^{-2} b^{-c}\right) \\
z & =\Omega\left(n^{-2} b^{-c}\right)^{1+1 /(\alpha-1)}
\end{aligned}
$$

At the same time, we know $z_{G, \alpha}(b) \geq b^{c}$ since the min-cut contributes. As one bound increases and one decreases with $b$, their crossing point where $b^{c}=\left(n^{-2} b^{-c}\right)^{1+1 /(\alpha-1)}$ gives a universal lower bound. Solving yields $b^{c}=n^{-1-1 /(2 \alpha-1)}$ as a lower bound 
for $z_{G, \alpha}(b)$. We conclude that $u_{G}(s)=\Omega\left(n^{-1-O(1 / \alpha)} \log ^{-2 \alpha} n\right)$ for every $\alpha$. In particular setting e.g. $\alpha=\log \log n$ gives $z \geq n^{-1-o(1)}$.

It follows that if we use NMC for $p>s$ and RCA for $p \leq s$, the bottleneck is NMC at $p=s$ which, with sparsification, will take $n^{2+o(1)}$ time. However, the $\log ^{\alpha} n$ factor in our analysis prevents our achieving an $\tilde{O}\left(n^{2}\right)$ bound. To do so, we generalize our argument to more than one intermediate sampling stage.

\subsection{Refinement}

We can strengthen the analysis to $z_{G}(s) \geq n^{-1 / 2-o(1)}$ by considering two intermediate graphs. Consider any $\alpha_{1}, \alpha_{2}$ such that $\alpha_{1}>\alpha_{2}$ and suppose that $z=z_{G, \alpha_{2}}(b)$ is small. Define $q_{1}=b /(8 z)^{1 / \alpha_{1} c}$ and $q_{2}=b /(8 z)^{1 / \alpha_{2} c}$ so $q_{2}<q_{1}$. Then define $H_{1} \sim G\left(q_{1}\right)$ and $H_{2} \sim G\left(q_{2}\right) \sim H_{1}\left(q_{2} / q_{1}\right)$ so that $G(b) \sim H_{2}\left(b / q_{2}\right)$. Arguing as above, we conclude that $H_{1}$ has likely size $h_{1} \leq n^{2} q_{1}^{c / 2}$ and mincut at least $\alpha_{1} c$; conditioning on this we argue the same way that $H_{2} \sim H_{1}\left(q_{2} / q_{1}\right)$ has likely min-cut at least $\alpha_{2} c$ and, since it is being generated from $H_{1}$ with larger min-cut $\alpha_{1} c$, likely size at most $h_{2} \leq h_{1}\left(q_{2} / q_{1}\right)^{\alpha_{1} c / 2}$. In this likely scenario, the probability $\mathrm{H}_{2}\left(b / q_{2}\right)$ is disconnected is at most

$$
\begin{aligned}
h_{2}^{2}\left(b / q_{2}\right)^{\alpha_{2} c} & \leq\left(h_{1}\left(q_{2} / q_{1}\right)^{\alpha_{1} c / 2}\right)^{2}\left(b / q_{2}\right)^{\alpha_{2} c} \\
& =h_{1}^{2}\left(q_{2} / q_{1}\right)^{\alpha_{1} c}\left(b / q_{2}\right)^{\alpha_{2} c} \\
& \leq n^{2} q_{1}^{c}\left(q_{2} / q_{1}\right)^{\alpha_{1} c}\left(b / q_{2}\right)^{\alpha_{2} c} \\
& =n^{2} \frac{b^{c}}{(8 z)^{1 / \alpha_{1}}} \cdot(8 z)^{\left(1 / \alpha_{1} c-1 / \alpha_{2} c\right) \alpha_{1} c} \cdot(8 z)^{\left(1 / \alpha_{2} c\right) \alpha_{2} c} \\
& =n^{2} \frac{b^{c}}{(8 z)^{1 / \alpha_{1}}}(8 z)^{1-\alpha_{1} / \alpha_{2}}(8 z) \\
& =n^{2} b^{c}(8 z)^{2-1 / \alpha_{1}-\alpha_{1} / \alpha_{2}} .
\end{aligned}
$$

Continuing as above, this must exceed $1 / 4$ in order to contribute sufficient additional failure probability to $u_{G}(b)$ beyond that of the "unlikely" outcomes. In particular if $\alpha_{1}=4$ and $\alpha_{2}=16$ we find $n^{2} b^{c}(8 z)^{3 / 2}>1 / 4$, meaning $z=\Omega\left(\left(n^{2} b^{c}\right)^{-2 / 3}\right)$. Since also $z \geq b^{c}$, the crossing point where $b^{c}=\left(n^{2} b^{c}\right)^{-2 / 3}$, meaning $b^{c}=$ $n^{-4 / 5}$, is a lower bound. Thus $z_{G, 16}(b)=\Omega\left(n^{-4 / 5}\right)$ so $u_{G}(s)=$ $\Omega\left(n^{-4 / 5} / \log ^{16} n\right)$. More generally, as the $\alpha_{i}$ grow large, we find $u_{G}(s)=n^{-1 / 2-o(1)}$. This suffices to prove that we need only run NMC when $u_{G}(p)=\Omega(1 / n)$, yielding our claimed $\tilde{O}\left(n^{2}\right)$ time bound.

\section{CONNECTIVITY IN CONTINUOUS TIME}

The previous section strengthened the phase transition analysis by chaining two stages of contraction. We can derive ever-stronger bounds by chaining ever-more stages, but in the limit we arrive at a different model of the graph evolving over time. This is a well known model in reliability theory, going back at least to the work of Lomonosov and Poleskii $[10,11]$. Using it, we prove the following:

Theorem 7.1. $z_{G, \alpha}(b)=n^{-O(1 / \log \alpha)}$.

A simple corollary follows:

Theorem 7.2. $u_{G}(s)=n^{-O(1 / \log \log n)}$.
Proof. Since $s^{c}=\Omega\left(b^{c} / \log ^{2} n\right)$, it follows that

$$
\begin{aligned}
z_{G, \alpha}(s) & =\Omega\left(z_{G, b}(s) / \log ^{2 \alpha} n\right) \\
& =n^{-O(1 / \log \alpha)} / \log ^{2 \alpha} n .
\end{aligned}
$$

Balancing the numerator $N=n^{-O(1 / \log \alpha)}$ and denominator $D=$ $\log ^{2 \alpha} n$ we set $D=1 / N$ and find $\alpha=O(\log n) /(\log \log n)^{2}$ which makes $N=n^{-O(1 / \log \log n)}$ and also $1 / D=n^{-O(1 / \log \log n)}$, implying $u_{G}(s)=\Omega\left(z_{G}(s)\right) \geq z_{G, \alpha}(s)=N / D=n^{-O(1 / \log \log n)}$.

This second theorem shows that we can switch to the RCA as soon as $u_{G}(s)<n^{-(O(1 / \log \log n))}$, meaning that (with sparsification) NMC will never take time exceeding $n^{1+o(1)}$. The runtime bottleneck is thus the RCA. We devote this section to proving the first theorem.

\subsection{The Evolutionary Model}

We model $G$ as evolving over time, with edge $e$ arriving at time $t_{e}$ exponentially distributed with mean 1 , so $\operatorname{Pr}\left[t_{e}>t\right]=e^{-t}$. Thus the graph $G^{*}(t)$ of edges that have arrived at time $t$ is equivalent to the graph $G(p)$ with $p=e^{-t}$. We contract the edges as they arrive, so $G$ shrinks over time. Some not-yet arrived edges become self loops due to contractions. The independent edge arrivals form a Poisson process. Thus at time $t$ when $M_{t}$ non-self-loop edges remain, the time until the next arrival of a non-loop edge to shrink $G^{*}$ is exponentially distributed with mean $M_{t}$. Equivalently, in the infinitesimal time interval $[t, t+d t]$, the probability of an arrival is $M_{t} d t$ where $M_{t}$ is the (instantaneous) arrival rate. If $G^{*}(t)$ has size $N_{t}$ and min-cut $c_{t}$ then $M_{t} \geq N_{t} c_{t} / 2$ is a lower bound on the arrival rate at time $t$. Each non-loop arrival decrements $N_{t}$, and $G^{*}$ becomes connected at the moment $t$ when $N_{t}<2$.

In our model the instantaneous rate is itself a stochastic process $N_{t} c_{t} / 2$. But suppose that instead the rate was $N_{t} r(t)$ for some deterministic unit rate function $r(t)$. Then an equivalent process, yielding the same distribution, is to start with $N_{0}=n$ distinct items and let $N_{t}$ be the number of items surviving at time $t$ as each "dies" independently in interval $[t, t+d t]$ with probability $r(t) d t$. The distribution is the same, since the probability some item dies in each interval is the requisite $N_{t} r(t) d t$. This model is known as a nonhomogeneous pure death process.

In this death process, the $n$ starting items all die independently. It thus suffices to know the survival probability $P_{t}$ of a single item at time $t$; we can then bound the disconnection probability at time $t$ by $\operatorname{Pr}\left[N_{t} \geq 2\right] \leq\left(\begin{array}{l}n \\ 2\end{array}\right) P_{t}^{2}$ by applying the union bound to the $\left(\begin{array}{c}n \\ 2\end{array}\right)$ possible pairs of surviving items. We can derive $P_{t}$ as the probability that the single item survives in every infinitesimal interval, which is

$$
\begin{aligned}
\lim _{d t \rightarrow 0} \prod_{0 \leq t \leq t^{\prime}}(1-r(t) d t) & =\lim _{d t \rightarrow 0} \prod_{0 \leq t \leq t^{\prime}} e^{-r(t) d t} \\
& =\lim _{d t \rightarrow 0} \exp \left(-\sum_{0 \leq t \leq t^{\prime}} r(t) d t\right) \\
& =\exp \left(-\int_{0}^{t^{\prime}} r(t) d t\right)
\end{aligned}
$$




\subsection{Analysis}

We now use the evolutionary model to relate $u_{G}(p)$ to $z_{G, \alpha}(p)$ and prove Theorem 7.1. Our analysis uses a conditioning event to reduce our problem to the case of a deterministic rate function.

As before, we assume $z=z_{G, \alpha}(p)$ is small for some $p$. Take $\pi=$ $\ln 1 / p$ to be the time in our evolutionary model $G^{*}(t)$ corresponding to edge failure probability $p$ in $G(p)$. At time $\pi$, a cut of value $c_{i}$ is still present with probability $e^{-c_{i} \pi}=p^{c_{i}}$. So the expected number of $\alpha$-minimum cuts surviving is $\sum_{c_{i} \leq \alpha c} e^{-c_{i} \pi}=z$.

Lemma 7.3. With probability $1-e^{\tau} z$, for all $t \leq \pi-\tau / \alpha c$, the minimum cut of $G^{*}(t)$ is at least $\tau /(\pi-t)$.

Proof. The probability that cut $i$ survives in $G^{*}\left(\pi-\tau / c_{i}\right)$ is $e^{-c_{i}\left(\pi-\tau / c_{i}\right)}=e^{-c_{i} \pi} e^{\tau}$. So the probability any $\alpha$-minimum cut $i$ survives to its corresponding time $\pi-\tau / c_{i}$ is union-bounded by $e^{\tau} \sum_{c_{i} \leq \alpha c} e^{-c_{i} \pi}=e^{\tau} z$. If none does, then the minimum cut at time $t=\pi-\tau / x$ is at least $x$, where $x=\tau /(\pi-t)$ as claimed.

Let $R$ be the event described in the lemma. If $R$ holds, we can define a lower bound $r(t)$ on the rate for all $t$ as follows. When $t \leq t_{1}=\pi-\tau / c$ we have lower bound $r(t)=c / 2$ since the minimum cut is always at least $c$. For $t_{1} \leq t \leq t_{\alpha}=\pi-\tau / \alpha c$ we have $r(t)=\tau / 2(\pi-t)$ per the lemma. And for $t>t_{\alpha}$ we have $r(t)=\alpha c / 2$ since per the lemma all $\alpha$-minimum cuts are gone by time $t_{\alpha}$.

While $R$ implies the rate lower bound $r(t)$, conditioning on $R$ biases the arrival of edges. To handle this, we define another process $B_{t}$ that is coupled to $N_{t}$. In the infinitesimal interval $[t, t+d t]$, the process $B_{t}$ decrements if $N_{t}$ does. But if at time $t$ small cuts remain and make the rate less than $N_{t} r(t) d t$, we decrement $B_{t}$ with additional probability sufficient to raise the total probability of a decrement in $B_{t}$ to $N_{t} r(t) d t$. Thus, the rate for $B_{t}$ is always at least $r(t)$, meaning it is stochastically dominated by a death process with that rate. At the same time, if $R$ holds then the extra decrement is never invoked, so $B_{t}=N_{t}$.

As discussed above, we can understand $\operatorname{Pr}\left[B_{\pi} \geq 2\right]$ through the probability that a single item, dying with rate function $r(t)$, survives to time $\pi$. As above, this is $e^{-L}$ where

$$
\begin{aligned}
L & =\int_{0}^{\pi} r(t) d t \\
& =\int_{0}^{t_{1}}(c / 2) d t+\int_{t_{1}}^{t_{\alpha}} \frac{\tau}{2(\pi-t)} d t+\int_{t_{\alpha}}^{\pi}(\alpha c / 2) d t \\
& =t_{1}(c / 2)-\frac{\tau}{2} \int_{\pi-t_{1}}^{\pi-t_{\alpha}} \frac{1}{x} d x+\left(\pi-t_{\alpha}\right)(\alpha c / 2) \\
& =(\pi-\tau / c)(c / 2)-\frac{\tau}{2} \ln \left(\frac{\tau / c}{\tau / \alpha c}\right)+(\tau / \alpha c)(\alpha c / 2) \\
& =(\pi c / 2-\tau / 2)+(\tau / 2) \ln (\alpha)+\tau / 2 \\
& =(\pi c / 2)+(\tau / 2) \ln \alpha .
\end{aligned}
$$

If we now write $\delta=e^{\tau} z$ to be the probability (per the lemma) that event $R$ does not hold, then the single-item survival probability is

$$
\begin{aligned}
e^{-L} & =e^{-\pi c / 2} \cdot e^{\tau(\ln \alpha) / 2} \\
& =p^{c / 2} \cdot(z / \delta)^{(\ln \alpha) / 2} \\
\text { since } e^{-\pi} & =p \text {. Therefore, } \operatorname{Pr}\left[B_{t} \geq 2\right] \leq n^{2} p^{c}(z / \delta)^{\ln \alpha} .
\end{aligned}
$$

We have analyzed $B_{\pi}$ while our target is $N_{\pi}$. But by definition these are identical so long as the event $R$ occurs. It follows that, $\operatorname{Pr}\left[\left(N_{\pi} \geq 2\right) \cap R\right]=\operatorname{Pr}\left[\left(B_{\pi} \geq 2\right) \cap R\right]$. We can therefore write

$$
\begin{aligned}
u_{G}(p) & =\operatorname{Pr}\left[N_{t} \geq 2\right] \\
& =\operatorname{Pr}\left[\left(N_{t} \geq 2\right) \cap R\right]+\operatorname{Pr}\left[\left(N_{t} \geq 2\right) \cap \bar{R}\right] \\
& \leq \operatorname{Pr}\left[\left(B_{t} \geq 2\right) \cap R\right]+\operatorname{Pr}[\bar{R}] \\
& \leq \operatorname{Pr}\left[B_{t} \geq 2\right]+\operatorname{Pr}[\bar{R}] \\
& \leq n^{2} p^{c} \cdot(z / \delta)^{\ln \alpha}+\delta
\end{aligned}
$$

if we now balance by choosing $\delta$ to equalize the two terms, we find

$$
u_{G}(p) \leq\left(n^{2} p^{c}\right)^{1 / \ln \alpha e} z^{1-1 / \ln \alpha e} .
$$

When $\alpha=1$, this simply recovers our original bound $u_{G}(p) \leq n^{2} p^{c}$. In the limit as $\alpha \rightarrow \infty$, we recover our other bound $u_{G}(p) \leq z_{G}(p)$. But we now have a bound that evolves with all $\alpha$ in between.

Next, consider the breakpoint $b$ where $u_{G}(b)=1 / 2$. Set $\delta=1 / 4$. We conclude that

$$
\begin{aligned}
1 / 2 & \leq n b^{c / 2}(4 z)^{\ln \alpha}+1 / 4 \\
\left(n b^{c / 2}\right)(4 z)^{\ln \alpha} & \geq 1 / 4 \\
z_{G, \alpha}(b) & =\left(n b^{c / 2}\right)^{-\Omega(1 / \ln \alpha)} \\
& \geq n^{-\Omega(1 / \ln \alpha)}
\end{aligned}
$$

This proves Theorem 7.1.

One can use similar techniques to derive stronger bounds on the expected size of $G(p)$, and also generalize to apply the bound on $z_{G, \alpha}(p)$ to $u_{G}(q)$ for $q \neq p$ by stopping the integration at $t=\ln 1 / q$ instead of $t=\ln 1 / p$.

\section{CONCLUSION}

This work has developed some new techniques for analyzing the ways cut failures interact, and through them tightened our understanding of the evolution of the failure probability $u_{G}(p)$ as $p$ varies. Based on this understanding, we have shown that so long as $p$ is smaller than the strongpoint of $G$, the unbiased estimator $u_{H}(p / q)$ has relative variance $\tilde{O}\left(q^{-c}\right)$, allowing the Recursive Contraction Algorithm to be applied to estimate $u_{G}(p)$ in $\tilde{O}\left(n^{2}\right)$ time. We have also shown that above the strongpoint $u_{G}(p)=n^{-o(1)}$, allowing us to apply Naive Monte Carlo sampling. Combining these two approaches yields an unbiased estimator algorithm for $u_{G}(p)$ with a runtime of $\tilde{O}\left(n^{2}\right)$.

As noted above, a graph with just a single minimum cut shows that the relative variance of $u_{H}(p / q)$ is at least $q^{-c}$. While we have now shown it to be $\tilde{O}\left(q^{-c}\right)$ when $p \leq s$, we conjecture that the $q^{-c}$ bound is the worst case and holds for all $p$. When there are more cuts, intuition suggests that we are adding random variables which should decrease the relative variance. This intuition fails when the variables are correlated, but our analysis above provides intuition that cut failures act reasonably independent of one another.

Should this universal $q^{-c}$ bound hold, the Recursive Contraction Algorithm would immediately yield an $\tilde{O}\left(n^{2}\right)$-time estimator for network reliability for all $p$. But that may not be the end. Graphs with many minimum cuts-i.e., unreliable graphs-have relative variance significantly smaller that $q^{-c}$, which would allow the RCA 
to run with fewer recursive calls and improve on its $\tilde{O}\left(n^{2}\right)$ time bound. Conversely, reliable graphs, with few minimum cuts, tend to shrink more quickly, which might allow the RCA to run to a shallower depth and again improve on the $\tilde{O}\left(n^{2}\right)$ time bound. We therefore conjecture that a near-linear-time algorithm exists.

\section{A UNBIASED ESTIMATORS}

We wish to estimate the probability $u_{G}(p)$ that $G$ becomes disconnected when edges fail with probability $p$ producing a random graph $G(p)$. If $u_{G}(p)$ is large we can do so by direct experiment, deleting edges and testing with $G$ is connected. This naive Monte Carlo (NMC) algorithm requires roughly $1 / u_{G}(p)$ trials for a good estimate.

When $u_{G}(p)$ is small NMC is too slow. In previous work [7] we derived a natural unbiased estimator for this problem. To construct $G(p)$, consider marking each edge for possible deletion with probability $q$, then deleting each marked edge with probability $p / q$. This deletes each edge with probability $(p / q) \cdot q=p$ as desired. But unmarked edges are never deleted, so the only cuts that can fail are those with all edges marked. Thus, we can contract all unmarked edges before the actual deletion step, yielding a graph $H$ distributed as a sample from $G(q)$-written as $H \sim G(q)$. The probability $u_{G}(p)$ that $G(p)$ is disconnected is then the probability $H(p / q)$ is disconnected, namely $u_{H}(p / q)$, meaning $u_{G}(p)=E_{H}\left[u_{H}(p / q)\right]$. In other words, $u_{H}(p / q)$ is an unbiased estimator for $u_{G}(p)$. This proves Lemma 1.1. This means that $u_{G}(p)$ can be estimated by repeatedly generating samples $H$ and averaging their reliabilities.

The key to this approach is bounding the relative variance of this estimator.

Definition A.1. The relative variance of random variable $X$ is $\frac{E\left[X^{2}\right]}{E[X]^{2}}-1$.

If the relative variance is $r$ then combining $O(r)$ samples yields an FPRAS. The following lemma is a well-known application of the Chebyshev bound.

Lemma A.2. If $X$ has relative variancer, then the median of $O(\log 1 / \delta)$ averages of $O\left(r / \epsilon^{2}\right)$ independent samples of $X$, totaling $O\left(r \epsilon^{-2} \log 1 / \delta\right)$ samples, yields an $\epsilon$ approximation to $E[X]$ (i.e., a value in the range $(1 \pm \epsilon) E[X])$ with probability $1-\delta$.

Corollary A.3. If an unbiased estimator for $X$ has relative variance $r$, then there is an FPRAS for $X$ using $O\left(r \epsilon^{-2} \ln (1 / \delta)\right)$ samples of the unbiased estimator.

\section{B RELATIVE VARIANCE ANALYSIS}

In this section we prove Lemma 3.2.

We wish to bound

$$
\frac{\sum\left(p^{2} / q\right)^{c_{i}}-\sum p^{2 c_{i}} / q^{c}}{\left(\sum p^{c_{i}}\right)^{2}}=q^{-c} \frac{\sum\left(\left(p^{2} / q\right)^{c_{i}-c}-p^{2\left(c_{i}-c\right)}\right)}{\left(\sum p^{c_{i}-c}\right)^{2}}
$$

Changing variables, write $\epsilon=\log _{p} q$ so that $q=p^{\epsilon}$. Furthermore, write $x_{i}=p^{c_{i}-c}$ so that $\left(p^{2} / q\right)^{c_{i}-c}=x_{i}^{2-\epsilon}$. Then our sum becomes

$$
=q^{-c} \sum \frac{x_{i}^{2-\epsilon}-x_{i}^{2}}{\left(\sum x_{i}\right)^{2}}
$$

Note that $x_{1}=1$.

We can bound this sum using standard calculus methods.

Lemma B.1. Assume all $x_{i} \geq 0$ and $x_{1}=1$. Then

$$
\frac{\sum\left(x_{i}^{2-\epsilon}-x_{i}^{2}\right)}{\left(\sum x_{i}\right)^{2}}=O(\epsilon)
$$

Note that if all $x_{i} \rightarrow 0$ then the ratio is unbounded like $x^{-\epsilon}$; the requirement that some $x_{i}=1$ prevents this.

Write $z=\sum x_{i}$ so the denominator is $z^{2}$. Write $f(x)=x^{2-\epsilon}-x^{2}$, so the numerator is $\sum f\left(x_{i}\right)$. Consider the shape of $f(x)$. It is 0 at 0 and 1 and positive between. It is negative for $x>1$, so no $x_{i}>1$ when our lemma's ratio is maximized. The first derivative $f^{\prime}(x) \geq 0$ iff

$$
\begin{aligned}
0 & \leq(2-\epsilon) x^{1-\epsilon}-2 x \\
2 x & \leq(2-\epsilon) x^{1-\epsilon} \\
x^{\epsilon} & \leq(1-\epsilon / 2) \\
x & \leq(1-\epsilon / 2)^{1 / \epsilon} \\
& \approx e^{-1 / 2}
\end{aligned}
$$

Thus, $f$ increases from 0 to a maximum at $x \approx e^{-1 / 2}$, then decreases. Its second derivative $f^{\prime \prime}(x) \geq 0$ iff

$$
\begin{aligned}
0 & \leq f^{\prime \prime}(x) \\
& =(2-\epsilon)(1-\epsilon) x^{-\epsilon}-2 \\
x^{\epsilon} & \leq(1-\epsilon / 2)(1-\epsilon) \\
x & \leq(1-\epsilon / 2)^{1 / \epsilon}(1-\epsilon)^{1 / \epsilon} \\
& \approx e^{-3 / 2}
\end{aligned}
$$

Thus, there is an inflection point at $w$ where $w^{\epsilon}=(1-\epsilon / 2)(1-\epsilon)=$ $1-O(\epsilon)$, and $f$ is concave below $w$ and convex above.

Consider first the concave region; if both $x_{i}$ and $x_{j}$ are in this region with $x_{i}<x_{j}$ then $f\left(x_{i}-\delta\right)+f\left(x_{j}+\delta\right) \geq f\left(x_{i}\right)+f\left(x_{j}\right)$ (since $f^{\prime}\left(x_{i}\right)<f^{\prime}\left(x_{j}\right)$ ). In other words, we can decrease $x_{i}$ and increase $x_{j}$ by the same amount and increase the sum. This change increases the numerator but leaves the denominator $z$ unchanged, so it strictly improves our bound. We can do this until either $x_{j}$ increases to the inflection point or $x_{i}$ decreases to 0 (and can be removed from the equation). It follows that at the maximum, there is at most one element $y$ in the concave region contributing $f(y)$. How much can it contribute? Since $f$ is increasing in the concave region (our computation above shows that $w$ is smaller than the maximizer of $f$ ) the maximum contribution is at $f(w)$. But

$$
\begin{aligned}
& f(w)=w^{2-\epsilon}-w^{2} \\
& =w^{2}\left(w^{-\epsilon}-1\right) \\
& =w^{2}\left((1-O(\epsilon))^{-1}-1\right) \quad \text { by definition of } w \\
& =w^{2} \cdot O(\epsilon) \\
& =O(\epsilon) \quad \text { since } w \leq 1
\end{aligned}
$$

Next we consider the $x_{i}$ in the convex region. Here, if any two $x_{i}$ are different then we can increase the numerator and leave the denominator $z$ unchanged by moving them towards each other. It follows that at maximum all $x_{i}$ in the convex region take on the same value $x$. Suppose there are $k$ such $x_{i}$ summing to $k f(x)$. Then 
differentiating by $k$ shows that the ratio $k f(x) / z^{2} \leq k f(x) /(1+$ $k x)^{2}=f(x) /\left(k^{-1 / 2}+x k^{1 / 2}\right)^{2}$ is maximized when $k=1 / x$ with value $f(x) / 4 x$. Given that $k$, we find the maximum of $f(x) / x=$ $x^{1-\epsilon}-x$ by setting the derivative with $x$ to 0 :

$$
\begin{aligned}
(1-\epsilon) x^{-\epsilon}-1 & =0 \\
x^{\epsilon} & =1-\epsilon \\
x & =(1-\epsilon)^{1 / \epsilon} \\
& \approx 1 / e
\end{aligned}
$$

and therefore

$$
\begin{aligned}
f(x) / x & =x\left(x^{-\epsilon}-1\right) \\
& =x\left((1-\epsilon)^{-1}-1\right) \\
& =O(\epsilon x) \\
& =O(\epsilon)
\end{aligned}
$$

Combining what we've done, we find that

$$
\begin{aligned}
\frac{\sum\left(f\left(x_{i}\right)\right)}{z^{2}} & =\frac{f(w)+k f(x)}{(1+w+k x)^{2}} \\
& \leq \frac{f(w)}{(1)^{2}}+\frac{k f(x)}{(1+k x)^{2}} \\
& =O(\epsilon)+O(\epsilon) \\
& =O(\epsilon) .
\end{aligned}
$$

\section{THE RECURSIVE ESTIMATOR}

Here, we give a slightly more detailed explanation of the recursive estimator algorithm discussed in Lemma 1.2. Full details can be found in our prior work [8].

We focus on the simpler case where the relative variance of $u_{H}(p / q)$ is at most $q^{-c}-1$ and in particular set $q^{c}=1 / 2$ so the relative variance is 1 . For now, assume have an oracle for whether $u_{G}(p) \leq n^{-\gamma}$. If it is not, then averaging $n^{\gamma}$ iterations of NMC, each taking $\tilde{O}\left(n^{1+\gamma}\right)$ time using sparsification, yields an unbiased estimator of relative variance 1 .

For smaller $u_{G}(p)$, we used the fact that $u_{H}(p / q)$ has relative variance 1 as an estimator for $u_{G}(p)$ and solved the problem recursively. We showed that if we generate $H \sim G(q)$ and then recursively construct an unbiased estimator of relative variance 1 for $u_{H}(p / q)$, we get an estimator of relative variance 2 for $u_{G}(p)$. But if we average two such recursive estimations, we can bring the relative variance back down towards 1 . We also know from Corollary 2.2 that $H$ will have size roughly $q^{c / 2} n=n / \sqrt{2}$. This gives us a runtime recurrence of the form

$$
\begin{aligned}
T(n) & \leq \max n^{1+\gamma}, 2 T(n \sqrt{2}) \\
& =O\left(n^{\max (2,1+\gamma)}\right)
\end{aligned}
$$

Finally, we must implement the oracle that decides which estimator algorithm to use at each recursion. First, if $p^{c} \leq n^{-2}$ then we know we should use the RCA unbiased estimator, while if $p^{c} \geq n^{-\gamma}$ we should use NMC. For the interval between, test by (separately) running $\tilde{O}\left(n^{\gamma}\right)$ iterations of NMC, taking time $\tilde{O}\left(n^{1+\gamma}\right)$, just to test whether $u_{G}(p) \leq n^{-\gamma}$. By setting constants correctly, we can get the correct answer with high probability and thus use the appropriate estimator with high probability. In the unlikely event that we apply the wrong estimator, it will still be a valid estimator, just with a higher relative variance. In particular, no matter what $q \geq n^{-2}$ we choose, we have $u_{H}(p / q) \leq n^{2}(p / q)^{c}$, while $u_{G}(p) \geq p^{c}$, which means the relative variance of our unbiased estimator is at most $\left(n^{2}(p / q)^{c}\right)^{2} / p^{2 c}=n^{O(1)}$. Since we use such a "bad" estimator with only polynomially small probability, its contribution to the overall relative variance is negligible.

\section{REFERENCES}

[1] Terje Aven and Uwe Jensen. 2013. Basic Reliability Theory. Springer New York, New York, NY, 17-55. https://doi.org/10.1007/978-1-4614-7894-2_2

[2] Charles J. Colbourn. 1987. The Combinatorics of Network Reliability. The International Series of Monographs on Computer Science, Vol. 4. Oxford University Press.

[3] Keith Frankston, Jeff Kahn, Bhargav Narayanan, and Jinyoung Park. 2019. Thresholds versus fractional expectation-thresholds. arXiv:arXiv:1910.13433

[4] Heng Guo and Mark Jerrum. 2019. A Polynomial-Time Approximation Algorithm for All-Terminal Network Reliability. SIAM f. Comput. 48, 3 (2019), 964-978. https://doi.org/10.1137/18M1201846

[5] David G. Harris and Aravind Srinivasan. 2017. Improved bounds and algorithms for graph cuts and network reliability. Random Structures \& Algorithms 52, 1 (2017), 74-135. https://doi.org/10.1002/rsa.20724 arXiv:https://onlinelibrary.wiley.com/doi/pdf/10.1002/rsa.20724

[6] David R. Karger. 2001. A Randomized Fully Polynomial Approximation Scheme for the All Terminal Network Reliability Problem. SIAM Rev. 43, 3 (2001), 499-522. A preliminary version appeared in Proceedings of the $27^{\text {th }}$ ACM Symposium on Theory of Computing. This corrects a version published in SICOMP.

[7] David R. Karger. 2016. A Fast and Simple Unbiased Estimator for Network (Un)reliability. In Proceedings of the 48th annual IEEE Symposium on Foundations of Computer Science (2016-10). 635-644.

[8] David R Karger. 2017. Faster (and still pretty Simple) Unbiased Estimators for Network (Un) reliability. In 2017 IEEE 58th Annual Symposium on Foundations of Computer Science (FOCS). IEEE, 755-766.

[9] David R. Karger and Clifford Stein. 1996. A New Approach to the Minimum Cut Problem. F. ACM 43, 4 (July 1996), 601-640. https://doi.org/10.1145/234533.234534 Preliminary portions appeared in SODA 1992 and STOC 1993.

[10] Micael V. Lomonosov. 1994. On Monte Carlo Estimates in Network Reliability. Probability in the Engineering and Informational Sciences 8 (1994), 245-264.

[11] Micael V. Lomonosov and V. P. Polesskii. 1971. Lower Bound of Network Reliability. Problems of Information Transmission 7 (1971), 118-123.

[12] Leslie Valiant. 1979. The Complexity of Enumeration and Reliability Problems. SIAM f. Comput. 8 (1979), 410-421. 